\title{
Specific antidote therapy in dogs: modern aspects
}

\author{
Svetlana Zykova ${ }^{1, *}$, Sergei Bogdanov ${ }^{1}$, Oleg Sukhanov ${ }^{1}$, Vyacheslav Dorofeev ${ }^{1}$ and \\ Roman Slobodyanik ${ }^{1}$ \\ ${ }^{1}$ Perm Military Institute of National Guard Troops, 614112, Perm, Russia
}

\begin{abstract}
Current laws of the Russian Federation provide for responsible treatment of animals. Nevertheless, there is an acute problem of the growing number of uncared for dogs. The article addresses issues of dog protection focused on the prevention of dog poisoning by medications illegally used by dog hunters, as well as on dog training aspects and methods of specific antidote therapy. Cytoprotective activity of ubiquinone is studied in view of biochemical profile in dogs.
\end{abstract}

\section{Introduction}

The most common problem that cynophiles and national security agencies' dog trainers encounter is canine unwanted behaviour when dogs try to eat various food residues, carrion, feces, which, in its turn, often leads to common food intoxications, infections caused by pathogenic microorganisms, helminths, and to the most dangerous situation when there is a risk of possible death of a dog due the consumption of intentionally poisoned food wastes as a result of illegal activity of "dog-hunters" ("dog-hunters" is a term standing for selfidentification of persons who, on their own, hunt or poison dogs in residential areas) [1].

Canine behaviour manifested as temptation to pick up and eat food residues and carrion is innate in predators and is genetically determined. Eating residues is caused, first of all, by the fact that raw rotten meat is fermented as a result of microbial contamination and is better and quicker digestible for animals.

Moreover, various perversions of the appetite in dogs may be caused by the development of the secondary lingual papillae hypotrophy, which appears in case of anemia (iron-deficient, macrocytic anemia) [2]. Feces of some animals, e.g. cattle, contain versatile microflora, which, in more favorable conditions, can produce a number of vitamins (for instance, E. coli synthesizes vitamin $\mathrm{B}_{12}$ ) and fibers that improve intestinal peristalsis in dogs.

In order to save life and health of a dog, you should preliminarily prevent exposure of the dog to various toxic compounds and thoroughly revise the dog's food ration. It is easier to deal with coprophagia. To do this, you should include all necessary nutrients, vitamins, minerals, Lactobacillus and Bifidobacterium in the dog's food ration. Brewers' yeast, Lactobacillus, Bifidobacterium, and tripe are additional sources of necessary microflora allowing you to feed the dog with all necessary nutrients and normalize its metabolism.

*Corresponding author: zykova.sv@rambler.ru 
In case of intoxication, timely administration of substances impeding hemolysis of red blood cells and antioxidants that block phospholipid peroxidation plays an important role. Particular attention is paid to antioxidants, because today there are actual data on the development of oxidative stress as one of key components of pathogenesis. The literature addresses different mechanisms of antioxidant activity development: antiradical mechanism, effect of compounds on antioxidant enzymes (superoxide dismutase and catalase), and membrane protection [3].

Studies, performed on the background of toxic agents of unclear etiology, demonstrated that the administration of multivitamin complexes containing nicotinic acid, vitamin $\mathrm{B}_{1}$, vitamin $B_{6}$ gives good chances to animals to survive. Most part of such compounds represents lipotropic factors of phospholipid synthesis in the liver, which are antioxidants by their chemical nature. We suppose that antioxidants can influence key enzymes of oxidation reduction hemostasis: superoxide dismutase, catalase, and glutathione. Such antioxidants include resveratrol which is a natural phytoalexin exhibiting antiradical activity and antioxidant activity in respect of sirtuin proteins (for instance, Sirtuin 1) [3].

\section{Materials and Methods}

In addition to a definite food ration, a prerequisite for the reduction of canine motivation to try to eat food residues exposed to insignificant microbiological deterioration and/or feces is the need to train a dog to refuse food which is not in its bowl and refuse food from a stranger.

Today, special remote-control electronic devices have proven to be the most efficient for dogs [4].

If such a device is used properly, a dog will never understand that the action is caused by a person and will associate all discomfort with exactly that "tasty" food that emits very «attractive» odour. The only particular feature of the use of such a device is the need to always have it on the dog each time you take the dog outside. If you put such device on the dog only for the time of trainings and do not put it on it when you walk the dog, the dog will quickly associate all negative feeling with the device and will be good at trainings, but you will never be able to deal with the problem of eating food residues by the dog when you walk the dog.

The action of electronic devices consists in electrical stimulus, vibration, and unpleasant sound produced.

In the beginning, you should use the electrical stimulus of the lowest power and gradually increase it to the level that causes discomfort and, respectively, refusal of scattered food exactly at the moment of occurrence of the motivation to pick up food (study).

After a learned reflex is developed and the dog refuses food after the minimum electrical stimulus, a vibration mode should be switched on, as the basic mode, and the vibration mode should be used together with the electrical stimulus for some period of time; then, everything should be gradually switched to the vibration mode only. Correct approach does not lead to any painful effect on a dog; it causes discomfort when the electrical stimulus is on, if the dog tries to eat food residues outside.

Depending on the features of an electronic device, along with the vibration mode you can use a strong sound signal.

Remote-control electronic devices are not cheap, so they are not always affordable.

Due to this, there is an alternative method which is based on the use of sound described by John Fisher in his book Think Dog. This method can be equally used in dog training, and the only disadvantage of this method compared to the abovementioned devices is that a dog 
trainer should assure timely effect on a dog at a distance and that there is need to make a lot of movements, at the same time, keeping more or less close to the dog.

John Fisher started using metal disks, which are now known as John Fisher Dog Training Discs.

The method of using the disks consists in the preliminary development of a learned reflex, which is done before dealing with a problem of any unwanted behaviour; therefore, a learned reflex has a necessary unpleasant action [4]. A dog trainer alternates the situations: first, he/she, letting the dog eat from his/her hand, pronounces an encouraging command that allows the action; then, the dog trainer puts some food on the floor and does not say anything, and, at the moment when the dog tries to pick up the food, the dog trainer starts using the disks making noises, and then the trainer throws the disks to the place where there is the food on the floor. Once the dog has moved aside, the disks and the food should be taken away. Later on, it is possible to give a treat to the dog, which refused food from the floor and moved aside.

There is also a method consisting in the use of a muzzle to walk the dog. First of all, it is important to correctly train the dog to wear the muzzle without any attempt to get rid of the muzzle, especially in the presence of a dog trainer.

The training starts with the use of different types of muzzles as stimulus to eat food offered by a dog trainer. To do this, in the beginning, the trainer uses a muzzle exceeding the size of the dog's snout and having an open frontal part through which the trainer let the dog eat from his/her hand.

Then, the time the muzzle is on the dog should be gradually increased, and the dog should be encouraged in places where it is taken for a walk. Once the dog can be easily taken for a walk with a semi-open muzzle on, the muzzle is replaced with a closed muzzle.

From 2016 through 2019, Perm Military Institute of National Guard Troops of the Russian Federation conducted a research to develop a canine habit to refuse to attack a butyl mercaptan-marked person who was wearing training clothes. To conduct the research, specialists selected dogs demonstrating acute predator's capturing behaviour manifested in strong motivation to attack a person. All dogs were trained to apprehend a person. The odour marker was attached to one of assistants wearing training clothes. The dogs were always allowed to attack an unmarked person. They were trained to ignore and refuse to attack a marked person.

One of the aspects studied during the research was finding the best way to exert an effect on a dog to train it to refuse to attack a marked person.

From the very beginning, the dogs were trained not to attack a marked person; that was achieved through the use of electrical stimulus, pinch collars, food, restraint leash, and muzzle. The marked and unmarked assistants were of the same height, body build, and had training clothes of the same shape and color.

All groups of dogs had a seven-day training pause after a week of training to refuse to attack in accordance with respective methods of training, and an experiment was performed to check attempts to attack a marked assistant without any influence exerted on dogs in one or another manner. All experimental findings were recorded.

Of course, if efforts focused on the prevention of picking up of toxicant-containing food by dogs turn out to be insufficient, a number of urgent measures shall be taken to prevent the progression of intoxication. One of the most important steps is to induce vomiting in the dog. To improve washing the stomach, sodium bicarbonate is used; it should be slightly warm to better induce vomiting reflex. The thing is that if some time pass from the moment of swallowing the bait (more than 2 hours) and convulsions begin, you should refuse to induce vomiting. The analysis of the situation with the administration of sorbents allows using physical adsorption (activated carbon which shall be preliminarily ground) and chemical adsorbents (silica gel, simeticone, diosmectite). The latter are more preferable if 
dogs have diarrhea. The next step is to choose a specific antidote therapy which should consist in the use of substances which can chemically bind to toxic substances (sodium thiosulfate solution can be used to bind to iodine; hydrogen peroxide solution can be used to bind to strong oxidizing agents: potassium permanganate or potassium bichromate).

The most important constituents of combined antidote therapy are vitamins (ascorbic acid reacts with iodine). For instance, vitamin PP (nicotinic acid, nicotinamide) and $\mathrm{B}_{6}$ (pyridoxine hydrochloride) are specific medications to treat isoniazid intoxication in dogs. The study shows that in case of isoniazid intoxication in dogs, massive hemolysis of red blood cells, hemodynamic compromise, cellular element failure, and stasis occur, thus leading to tubulonephrosis and hyperemia in the liver $[5,6]$.

A particular group of substances leading to intoxications includes pesticides, which represent various heterocyclic compounds. If pesticides get into the body of a dog, the following symptoms of intoxications develop: gastrointestinal atony, tachycardia, dyspnea, and convulsions. Acute intoxication pathomorphological manifestations include passive congestion of the liver, kidneys, and spleen, acute dilatation of the stomach and blind gut; in case of chronic intoxication caused by imidacloprid-containing pesticides, hydropic degeneration is observed in the liver and kidneys; in case of intoxication caused by cyanocontaining neonicotinoids, cloudy swelling, leukocyte infiltration, and connective tissue growth are observed [7]. It has been demonstrated that combined administration of unitiol in the amount of $0,025 \mathrm{~g} / \mathrm{kg}$ and diazepam in the amount of $0,5 \mathrm{mg} / \mathrm{kg}$ helps to accelerate the biotransformation of pesticides and leads to the reduction their residual amount concentration in the body of a dog and increases the survival of animals up to $75-80 \%$ [6].

A group of substances causing severe intoxications in animals include anticoagulant rodenticides which are used in rodent control. If such substances get into the body of a dog, they cause severe symptoms. General depression of an animal is observed; as a rule, rectal temperature is normal; visible mucous membranes are anemic; multiple hemorrhages in the mucous membrane are seen on the posterior pharyngeal wall and internal surface of cheeks. At the moment of auscultation, no noises are detected; the breathing is easy, chestabdominal. Hemorrhages in joints are observed; such joints are painful at palpation [8,9].

We have studied a number of antioxidant compounds which can be used as cytoprotective and antihypoxic drugs. Ubiquinone is one of such substances. We studied the use of ubiquinone in dogs throughout a period of 90 days. We conducted tests that included the observation of peripheral blood and blood chemistry. We assumed that in case of intoxication accompanied with hemolysis and hepatosis, we needed substances which could be used as specific antioxidants - resveratrol and ubiquinone - to bind to radicals formed in lipid peroxidation $[10,11,12]$.

We studied ubiquinone influence on canine blood chemistry in German Shepherds (the total number of animals was 30). Ubiquinone was administrated to the dogs in the amount of $15 \mathrm{mg}$ per $10 \mathrm{~kg}$ of weight of an animal during morning feeding throughout a period of 90 days. The group of dogs included 30 animals aged 5 to 9 years, 26 of which were males and 4 females.

\section{Results}

The results of the research, focused on studying various methods of exerting effect on a dog in order to make it form a habit to refuse to attack a marked person, showed reliable stability when electrical stimulus was used. Each dog in the test group, where an electrical stimulus was used, demonstrated at least 25 attacks stopped at the discretion of a dog out of 30 attempts to attack a marked person; it corresponds to 83,3 percent of a positive effect of an electrical stimulus. The effectiveness of other stimuli did not exceed $25 \%$. 
Please see canine blood chemistry values before and after the use of ubiquinone (table 1).

Table 1. Canine blood chemistry values before and after the use of ubiquinone.

\begin{tabular}{|c|c|c|c|}
\hline \multirow{2}{*}{ Parameter } & \multirow{2}{*}{ Reference value } & \multicolumn{2}{|c|}{ Values } \\
\cline { 3 - 4 } & & before the use & after the use \\
\hline total protein & $40-73 \mathrm{~g} / \mathrm{L}$ & $55.94 \pm 5.89^{*}$ & $70.66 \pm 6.15^{*}$ \\
\hline glucose & $4.3-7.3 \mathrm{mmol} / \mathrm{L}$ & $3.18 \pm 0.81^{*}$ & $4.94 \pm 1.24^{*}$ \\
\hline ALT & $9-52 \mathrm{U} / \mathrm{L}$ & $47.82 \pm 3.53^{*}$ & $44.60 \pm 3.18^{*}$ \\
\hline AST & $11-42 \mathrm{U} / \mathrm{L}$ & $47.30 \pm 2.78$ & $42.34 \pm 2.42$ \\
\hline alkaline & $18-70 \mathrm{U} / \mathrm{L}$ & $62.54 \pm 2.47$ & $34.96 \pm 2.70^{*}$ \\
\hline phosphatase & $26-120 \mu \mathrm{mol} / \mathrm{L}$ & $75.80 \pm 8.32^{*}$ & $101.00 \pm 5.25^{*}$ \\
\hline creatinine & $685-2155 \mathrm{U} / \mathrm{L}$ & $575 \pm 19.77^{*}$ & $1316.4 \pm 25.5$ \\
\hline alpha-amylase & $3.5-9.2 \mathrm{U} / \mathrm{L}$ & $5.76 \pm 1.15$ & $6.68 \pm 1.67$ \\
\hline urea & $3-13.5 \mu \mathrm{mol} / \mathrm{L}$ & $7.46 \pm 1.87$ & $4.56 \pm 1.29$ \\
\hline bilirubin & &
\end{tabular}

Note: ${ }^{*}$ - statistically significant values at $\mathrm{p}<0,05$ compared to control values.

\section{Discussion}

In the process of the development of a stable acquired reflex in a dog to make the dog refuse to eat scattered food, a dog trainer often make mistakes. The most common mistake is the use of fresh food during dog trainings. As a result, dogs clearly understand that eating food outside leads to unpleasant feelings, whereas rotten meat will still attract animals and will break all acquired barriers in their behaviour.

Therefore, in addition to fresh food, dog trainers should use various residues, rotten meat, fish, and food, meat and fish products, i.e. everything that a dog can find outside being strongly attracted.

Moreover, a dog master makes a very big mistake when he/she approaches his/her dog in order to punish the dog for too strong interest in or eating food. Most of these methods are used too late: when the dog is already in the middle of doing something unwanted or, which is more frequent, when the dog has already done something unwanted [3]. The dog quickly associates the approaching person with an unpleasant feeling and starts to increase the distance. The punishment comes directly from the dog master, and the dog quickly understands that first it must do what it wants and then keep out of the master [3].

Hence ubiquinone has a positive effect on the physiological status of dogs; it does not impair, instead, it significantly improves some parameters of the blood chemistry.

As a result of the use of ubiquinone throughout a period of 90 days, a certain change in parameters of canine blood chemistry values was observed; in particular, total protein value, glucose, and urea increased, ALT and alkaline phosphatase were normalized. Positive changes demonstrated the improvement of metabolic processes and cytoprotective action that manifested in the normalization of marker enzymes in the liver.

\section{Conclusions}


In the literature and Internet you can find classical methods of developing a canine habit to refuse food, but such methods do not solve the problem as a whole; in such case, methods based on negative reward can be of help [3].

At the moment of preparing a dog to refuse food when you use an electronic device, all effect exerted on a dog should be remote and should not be associated with the master's behaviour in any way in order to avoid the formation of a conditional association of the human behaviour type with negative effect on a dog. Of course, according to the conducted experiment (focused on dogs' refuse to attack an odour-marked assistant wearing training clothes), the method based on the use of an electrical stimulus to form a canine habit to refuse scattered food is efficient, quick, and convenient to a dog trainer.

If you follow John Fisher's method, you need to know that you can use other objects as alternative to the discs, allowing you to perform actions resulting in a strong high pitched metallic sound.

In his practical work, John Fisher found that animals are more susceptible to a strong ringing sound which you can use to catch their attention and interrupt unwanted behaviour.

The most important thing in the use of such objects is their small size, distinctness of the sound, and occurrence the sound only when necessary. If you always use the device that produces an unpleasant metallic sound, the animal will quickly get accustomed to it, and, at the moment of unwanted behaviour, e.g. eating of food when it is prohibited, the action of the device will be reduced to a minimum.

The best all-in-one solution is considered to be the use of a muzzle. It is used when there is no chance to timely exert any effect by means of an electrical stimulus or soundproducing device. This happens, first of all, when a dog goes far from a dog trainer or to places where actions of an animal cannot be visually observed.

In case of intoxication caused by hepatotropic toxicants, the use specific antidotes, which can block lipid peroxidation, impedes the development of alteration and promotes cytoprotective activity. Such antidotes include hepatoprotective and antioxidant drugs: ubiquinone and resveratrol. We suggest oral administration of ubiquinone and resveratrol, as cytoprotective agents, in the amount of $15 \mathrm{mg}$ per $10 \mathrm{~kg}$ of weight of a dog as treatment of intoxication caused by unknown toxicants.

\section{References}

1. Á. Miklósi, B. Turcsán, E. Kubinyi, Elsevier Science, Burlington, 191-222 (2014) https://doi.org/10.1016/B978-0-12-407818-5.00007-3

2. S. Chikazawa, M.D. Dunning, J. Small Anim Pract. 57(7), 348-53 (2016) doi: 10.1111/jsap. 12498

3. Y.T. Wu, Free Radical Research 48 (9), 1070-1084 (2014)

4. L. Menchetti, C. Righi, G.Guelf, C. Enas, L.Moscati, S. Mancini, S. Dierio, Applied Animal Behaviour Science 213, 107-116 (2019) https://doi.org/10.1016/j.applanim.2019.02.008

5. D. Grimm, Science 348(6232), 274-279 (2015) DOI: 10.1126/science.348.6232.274

6. E. Mora, G.M. Fonseca, P. Navarro, A. Castano, J. Lucena, Journal of Veterinary Behvior 25, 76-84 (2018) https://doi.org/10.1016/j.jveb.2018.03.011

7. H.I. Kotsyumbas, N.P. Vretsona, Scientific and Technical Bulletin of State Scientific Research Control Institute of Veterinary Medical Products and Fodder Additives and Institute of Animal Biology 20(2), 238-246 https://doi.org/10.36359/scivp.2019-20-2.30 
8. A. Anadon, M.R. Martínez-Larrañaga, I. Ares, M.A. Martínez, Biomarkers in Toxicology (Second Edition), 655-691 (2019) https://doi.org/10.1016/B978-0-12814655-2.00038-4

9. L.K. Gerunova, V.I. Okolelova, Agricultural Biology 4, 75-78 (2013)

10. L.G. P'yanova, L.K.Gerunova, M.S. Drozdetskaya, T.V. Gerunov, Russian Journal of Applied Chemistry 92(8), 1122-1127 (2019)

11. BSAVA/VPIS Guide to Common Canine and Feline Poisons (British Small Animal Veterinary Association, 2012) ISBN: 978- 1905319459

12. M. Prosek, J. Butinar, B.Lukanc, M. Milivojevic, L. Milivojevic, M. Krizman, A. Smidovnik, Journal of Pharmaceutical and Biomedical Analysis 47(4-5), 918-922 (2018) 